\title{
The O-glycan pathway is associated with in vitro sensitivity to gemcitabine and overall survival from ovarian cancer
}

\author{
NADIM BOU ZGHEIB $^{1}$, YIN XIONG ${ }^{1,2}$, DOUGLAS C. MARCHION ${ }^{1,2}$, ELONA BICAKU ${ }^{1,2}$, HYE SOOK CHON $^{1}$, \\ XIAOMANG BA STICKLES ${ }^{1}$, ENTIDHAR AL SAWAH ${ }^{1}$, PATRICIA L. JUDSON ${ }^{1,4}$, ARDESHIR HAKAM ${ }^{3,4}$, \\ JESUS GONZALEZ-BOSQUET ${ }^{1,4}$, ROBERT M. WENHAM ${ }^{1,2,4}$, SACHIN M. APTE ${ }^{1,4}$, \\ CHRISTOPHER L. CUBITT ${ }^{5}$, DUNG TSA CHEN ${ }^{6}$ and JOHNATHAN M. LANCASTER ${ }^{1,2,4}$ \\ ${ }^{1}$ Department of Women's Oncology, ${ }^{2}$ Experimental Therapeutics Program, ${ }^{3}$ Department of Anatomic Pathology, \\ ${ }^{4}$ Department of Oncologic Sciences at the University of South Florida, ${ }^{5}$ Translational Research and ${ }^{6}$ Biostatistics Core, \\ H. Lee Moffitt Cancer Center and Research Institute, Tampa, FL 33612, USA
}

Received January 9, 2012; Accepted March 16, 2012

DOI: $10.3892 /$ ijo.2012.1451

\begin{abstract}
Ovarian cancer (OVCA) is the most lethal gynecological malignancy. The high mortality rate associated with this disease is due in large part to the development of resistance to chemotherapy; however, the biological basis of this remains unclear. Gemcitabine is frequently used for the treatment of patients with platinum-resistant OVCA. We report molecular signaling pathways associated with OVCA response to gemcitabine. Forty-one OVCA cell lines were subjected to gene expression analysis; in parallel, $\mathrm{IC}_{50}$ values for gemcitabine were quantified using CellTiter-Blue viability assays. Pearson's correlation coefficients were calculated for gene expression and gemcitabine $\mathrm{IC}_{50}$ values. The genes associated with gemcitabine sensitivity were subjected to pathway analysis. For the identified pathways, principal component analysis was used to derive pathway signatures and corresponding scores, which represent overall measures of pathway expression. Expression levels of the identified pathways were then evaluated in a series of clinicogenomic datasets from 142 patients with stage III/IV serous OVCA. We found that in vitro gemcitabine sensitivity was associated with expression of 131 genes $(\mathrm{p}<0.001)$. These genes include significant representation of three molecular signaling pathways $(\mathrm{p}<0.02)$ : O-glycan biosynthesis, Role of Nek in cell cycle regulation and Antiviral actions of Interferons. In an external clinico-genomic OVCA dataset $(n=142)$, expression of the O-glycan pathway was associated with overall survival, independent of surgical cytoreductive status, grade and age
\end{abstract}

Correspondence to: Dr Johnathan M.Lancaster, Center for Women's Oncology, Department of Women's Oncology, Moffitt Cancer Center, 12902 Magnolia Drive, Tampa, FL 33612, USA

E-mail: johnathan.lancaster@moffitt.org

Key words: gemcitabine chemosensitivity, genomic study, O-glycan pathway, principal component analysis, ovarian cancer survival $(\mathrm{p}<0.001)$. Expression levels of Role of Nek in cell cycle regulation and Antiviral actions of Interferons were not associated with survival ( $p=0.31$ and $p=0.54$, respectively). Collectively, expression of the O-glycan biosynthesis pathway, which modifies protein function via post-translational carbohydrate binding, is independently associated with overall survival from OVCA. Our findings shed light on the molecular basis of OVCA responsiveness to gemcitabine and also identify a signaling pathway that may influence patient survival.

\section{Introduction}

Ovarian cancer (OVCA) is the leading cause of gynecologic cancer mortality and the sixth most common cancer diagnosed in women in the United States. Advanced-stage epithelial OVCA is highly heterogeneous at a clinical, biologic, and genetic level, but patients are currently treated in a uniform fashion with cytoreductive surgery and platinum/taxane-based combination chemotherapy. Unfortunately, most patients ultimately succumb to persistent or recurrent platinum-resistant disease $(1,2)$. Currently, efforts to develop therapeutic agents with greater efficacy against platinum-resistant disease are limited because of an incomplete understanding of the molecular determinants of OVCA drug response.

Gemcitabine (2',2'-difluorodeoxycytidine), a synthetic nucleoside analog of cytidine, is frequently used as a second-line therapy for patients with relapsed OVCA (3). As a pyrimidine analogue, gemcitabine replaces the nucleic acid cytidine during DNA replication, blocking processing and chain elongation by the DNA polymerase complex, resulting in G1 arrest and a subsequent cytostatic effect. Additionally, the gemcitabine triphosphate metabolite is incorporated into RNA, thus inhibiting RNA synthesis (4). Gemcitabine efficacy has been evaluated extensively both in vitro and in vivo against OVCA (5-8). Gemcitabine has demonstrated single-agent activity against OVCA cell lines (9) and synergistic activity with several other antineoplastic agents, including platinum compounds, topotecan, and etoposide (10). In animal tumor models, the gemcitabine effect has been shown to be schedule dependent, and continuous infusions over $24 \mathrm{~h}$ 
appear to enhance gemcitabine cytotoxicity (11). Phase II and III studies of gemcitabine $\left(800-1250 \mathrm{mg} / \mathrm{m}^{2} /\right.$ week) in patients with recurrent OVCA have demonstrated response rates up to $19 \%$ (12-14). Despite such data, the molecular determinants of gemcitabine activity remain to be fully elucidated. In this study, we sought to determine the molecular underpinnings of OVCA response to gemcitabine at a genome-wide level. We investigated the genes and molecular signaling pathways associated with the response of OVCA cells in vitro to gemcitabine and explored how these pathways influence in vivo clinical outcomes for patients with this disease.

\section{Materials and methods}

Overview. We subjected 41 OVCA cell lines to gene expression analysis and, in parallel, measured gemcitabine sensitivity $\left(\mathrm{IC}_{50}\right)$. Genes associated with baseline gemcitabine sensitivity, identified by Pearson's correlation analysis, were subjected to molecular pathway analysis. We evaluated expression of identified pathways using a series of clinico-genomic datasets from 142 patients with stage III/IV serous OVCA. All 142 patients had signed IRB-approved, written informed consent forms.

Cell culture. OVCA cell lines were obtained from the American Type Culture Collection (Manassas, VA; CAOV3, OV90, OVCAR3, SKOV3), from the European Collection of Cell Cultures (Salisbury, UK; A2780CP, A2780S), from Kyoto University (Kyoto, Japan; CHI, CHIcisR, M41, M41CSR, Tyknu, and TyknuCisR), or as kind gifts from Dr Patricia Kruk, Department of Pathology, College of Medicine, University of South Florida, Tampa, FL, and Susan Murphy, PhD, Department of OBGYN/Division of GYN Oncology, Duke University, Durham, NC (A2008, C13, CAOV2, HeyA8, IGR-OV1, IMCC3, IMCC5, MCAS, OV2008, OVCA420, OVCA429, OVCA432, OVCA433, FUOV1, PEO1, PEO4, SK-OV-6, T8, TOV-112D, TOV-21-G, Dov13, BG1, Ovary1847, OVCAR10, OVCAR8, OVCAR5, OVCAR4, OVCAR2, SK-OV-4). Cell lines were maintained in RPMI-1640 medium (Invitrogen, Carlsbad, CA) supplemented with $10 \%$ fetal bovine serum (Fisher Scientific, Pittsburgh,PA), $1 \%$ sodium pyruvate, $1 \%$ penicillin/streptomycin (Cellgro, Manassas, VA), and $1 \%$ non-essential amino acids (HyClone, Hudson, NH). Mycoplasma testing was performed every 6 months, in accordance with the manufacturer's protocol (Lonza, Rockland, ME).

RNA extraction and microarray expression analysis. RNA from 41 OVCA cell lines was extracted using the RNeasy kit following manufacturer's recommendations (Qiagen, Valencia, CA). Quality of the RNA was measured using an Agilent 2100 Bioanalyzer. The targets for Affymetrix DNA microarray analysis were prepared according to the manufacturer's instructions, and targets were hybridized to customized Human Affymetrix HuRSTA gene chips (HuRSTA-2a520709), which include 60,607 probe sets and representation of 19,308 genes (Gene Expression Omnibus accession number GSE34615).

CellTiter-Blue cell viability assays. Drug activity was evaluated using a high-throughput CellTiter-Blue cell viability assay. Cells $\left(2.5 \times 10^{3}\right.$ per well) were plated in 384 -well plates using complete media with $10 \%$ fetal bovine serum and allowed to adhere overnight. After cell adherence, increasing concentrations of gemcitabine were added to appropriate wells using an automated pipetting station. Four replicate wells were used for each drug concentration and for vehicle controls. Drug dilutions initially consisted of 1.5 -fold serial dilutions from a maximum concentration of $100 \mu \mathrm{M}$. The cells were incubated with the drug for $72 \mathrm{~h}$, and $5 \mu \mathrm{l}$ of CellTiter-Blue reagent (Promega Corp.) were added to each well. Fluorescence was read at 579-nm excitation/584-nm emission using a Synergy 4 microplate reader (Bio-Tek Instruments, Inc., Winooski, VT). $\mathrm{IC}_{50}$ values were determined using a sigmoidal equilibrium model fit (XLfit 5.2, ID Business Solutions Ltd.). The $\mathrm{IC}_{50}$ was defined as the concentration of drug required for a $50 \%$ reduction in growth/viability.

Statistical analysis. Expression data from 41 OVCA cell lines were subjected to background correction and normalization using the 'Robust Multichip Average' algorithm in the Affymetrix Expression Console (http://www.affymetrix.com/estore/index. jsp). Pearson's correlation test was performed on individual gene expression and $\mathrm{IC}_{50}$ values. Probe sets with $\mathrm{p}<0.001$ were considered to have significant correlations with $\mathrm{IC}_{50}$ values and were uploaded to MetaCore GeneGo for pathway analysis (http:// www.genego.com/metacore.php). Pathways with $\mathrm{p}<0.05$ were considered significant, based upon the GeneGo/MetaCore ${ }^{\mathrm{TM}}$ statistical test for significance.

Building signatures of pathway activity. The principal component analysis (PCA) methodology was used to derive a gene expression signature for each pathway. A corresponding 'pathway score' was thus generated that quantifies the overall level of pathway gene expression in a summary value. That is, the PCA score is a numeric value that summarizes the level of expression of the entire pathway. First, data were reduced into a small set of uncorrelated principal components. This set of principal components was generated based on its ability to account for variation. The first PCA was used to represent the overall expression level for the pathway as it accounts for the largest variability in the data. That is, the pathway score is equal to $\sum w_{i} x_{i}$, a weighted average expression among the pathway genes, where $x_{i}$ represents gene $i$ expression level, $w_{i}$ is the corresponding weight (loading coefficient) with $\sum w_{i}^{2}=1$, and the $w_{i}$ values maximize the variance of $\sum w_{i} x_{i}$. Details of this methodology have been previously reported by our group $(15,16)$.

Validation of signatures in primary OVCA datasets. The pathway gene expression signature scores were evaluated in an independent publicly available clinico-genomic dataset from 142 OVCA samples (16). In brief, all 142 samples were known to have been resected from patients with advanced-stage (III/IV), serous epithelial OVCA, who underwent primary cytoreductive surgery followed by primary therapy with a platinum-based regimen (+/- taxane or cyclophosphamide). Response to this primary therapy [complete response (CR) versus incomplete response (IR)] has previously been described for these patients (16). In brief, patients who demonstrated a CR had no evidence of disease on physical examination, serum tumor marker monitoring, or radiographic imaging. The IR category included all other patients. Log-rank tests with Kaplan-Meier survival 
Table I. Gemcitabine $\mathrm{IC}_{50}$.

\begin{tabular}{|c|c|c|c|}
\hline Cell line & $\mathrm{IC}_{50}($ mean$)$ & $\mathrm{IC}_{50}(\mathrm{SD})$ & No. of cells \\
\hline A2008 & $163.9 \mathrm{E}-9$ & $309.0 \mathrm{E}-9$ & 12 \\
\hline A2780CP & $366.4 \mathrm{E}-9$ & $696.6 \mathrm{E}-9$ & 9 \\
\hline A2780S & $51.8 \mathrm{E}-9$ & 46.5E-9 & 4 \\
\hline BG1 & $30.4 \mathrm{E}-6$ & $27.6 \mathrm{E}-6$ & 8 \\
\hline C13 & 418.1E-9 & $804.0 \mathrm{E}-9$ & 9 \\
\hline CAOV2 & $3.9 \mathrm{E}-6$ & $8.5 \mathrm{E}-6$ & 12 \\
\hline CAOV3 & $2.2 \mathrm{E}-9$ & $1.2 \mathrm{E}-9$ & 5 \\
\hline CHI & $268.6 \mathrm{E}-9$ & $522.6 \mathrm{E}-9$ & 26 \\
\hline CHIcisR & 23.7E-9 & $58.8 \mathrm{E}-9$ & 13 \\
\hline Dov13 & $6.0 \mathrm{E}-9$ & $3.5 \mathrm{E}-9$ & 4 \\
\hline FUOV1 & $59.4 \mathrm{E}-6$ & $6.9 \mathrm{E}-6$ & 3 \\
\hline HeyA8 & $1.5 \mathrm{E}-6$ & $2.4 \mathrm{E}-6$ & 6 \\
\hline IGR-OV1 & $531.2 \mathrm{E}-9$ & $1.3 \mathrm{E}-6$ & 14 \\
\hline IMCC3 & $942.6 \mathrm{E}-9$ & $1.1 \mathrm{E}-6$ & 15 \\
\hline IMCC5 & $105.1 \mathrm{E}-9$ & $159.6 \mathrm{E}-9$ & 20 \\
\hline M41 & $39.5 \mathrm{E}-9$ & 18.7E-9 & 5 \\
\hline M41CSR & $37.4 \mathrm{E}-9$ & 34.4E-9 & 12 \\
\hline MCAS & $56.4 \mathrm{E}-6$ & $99.8 \mathrm{E}-6$ & 8 \\
\hline OV2008 & $383.8 \mathrm{E}-9$ & $1.1 \mathrm{E}-6$ & 15 \\
\hline OV90 & 18.9E-9 & $11.9 \mathrm{E}-9$ & 9 \\
\hline Ovary1847 & $864.6 \mathrm{E}-9$ & $2.7 \mathrm{E}-6$ & 19 \\
\hline OVCA420 & $12.7 \mathrm{E}-6$ & 22.0E-6 & 5 \\
\hline OVCA429 & $22.2 \mathrm{E}-9$ & 24.2E-9 & 5 \\
\hline OVCA432 & $14.9 \mathrm{E}-6$ & 25.7E-6 & 3 \\
\hline OVCA433 & 9.9E-9 & 10.9E-9 & 5 \\
\hline OVCAR10 & $671.9 \mathrm{E}-9$ & $2.5 \mathrm{E}-6$ & 16 \\
\hline OVCAR2 & $22.0 \mathrm{E}-9$ & $30.8 \mathrm{E}-9$ & 17 \\
\hline OVCAR3 & $6.2 \mathrm{E}-6$ & $14.0 \mathrm{E}-6$ & 14 \\
\hline OVCAR4 & $655.3 \mathrm{E}-9$ & $870.8 \mathrm{E}-9$ & 5 \\
\hline OVCAR5 & $278.3 \mathrm{E}-9$ & $721.8 \mathrm{E}-9$ & 17 \\
\hline OVCAR8 & $272.6 \mathrm{E}-9$ & $681.6 \mathrm{E}-9$ & 12 \\
\hline PEO1 & $134.7 \mathrm{E}-9$ & $244.5 \mathrm{E}-9$ & 9 \\
\hline PEO4 & $536.2 \mathrm{E}-9$ & $868.6 \mathrm{E}-9$ & 10 \\
\hline SK-OV-3 & $16.1 \mathrm{E}-6$ & $30.5 \mathrm{E}-6$ & 11 \\
\hline SK-OV-4 & 3.3E-9 & $1.6 \mathrm{E}-9$ & 12 \\
\hline SK-OV-6 & $3.5 \mathrm{E}-6$ & $10.4 \mathrm{E}-6$ & 11 \\
\hline T8 & $255.6 \mathrm{E}-9$ & $456.6 \mathrm{E}-9$ & 9 \\
\hline TOV-112D & $44.5 \mathrm{E}-6$ & $67.3 \mathrm{E}-6$ & 9 \\
\hline TOV-21G & $764.1 \mathrm{E}-9$ & $1.5 \mathrm{E}-6$ & 11 \\
\hline Tyknu & 4.6E-9 & $2.8 \mathrm{E}-9$ & 4 \\
\hline TyknuCisR & $8.5 \mathrm{E}-9$ & $8.4 \mathrm{E}-9$ & 8 \\
\hline
\end{tabular}

curves were used to test any association between the pathway scores ('high' versus 'low' based on a median value cut-off) and overall survival for patients with OVCA.

\section{Results}

Forty-one OVCA cell lines were treated with increasing concentrations of gemcitabine, and the $I C_{50}$ values were determined (Table I). Pearson's correlation test using gemcitabine $\mathrm{IC}_{50}$ and OVCA cell line gene expression data identified 131 unique genes to be associated with gemcitabine sensitivity $(\mathrm{p}<0.001$; Table II). GeneGo MetaCore ${ }^{\mathrm{TM}}$ analysis identified three biological pathways that were represented within the 131 genes associated with gemcitabine sensitivity $(\mathrm{p}<0.02)$. These molecular signaling pathways included O-glycan biosynthesis $(\mathrm{p}=0.001)$, Role of Nek in cell cycle regulation $(\mathrm{p}=0.005)$, and Antiviral actions of Interferons $(\mathrm{p}=0.01)$. Statistical significance was derived from the total number of genes input into the pathway analysis software, the number of input genes present in a specific pathway, and the actual number of genes in that pathway. Thus, the p-value represents the probability that mapping a set of genes to a particular pathway occurs by chance. The O-glycan pathway demonstrated the highest level of statistical significance in its association with sensitivity to gemcitabine $(\mathrm{p}=0.001)$ (Fig. 1).

Expression of the O-glycan pathway is associated with OVCA clinical outcome. Based on the above findings, we utilized PCA to develop gene expression signature scores for the pathways associated with gemcitabine sensitivity in vitro (15). In this way, a 34-gene 'O-glycan biosynthesis pathway signature' (OGBPS) (Table III) was generated and evaluated in an independent OVCA genomic dataset (16). Using the median value as a threshold to define high versus low OGBPS score, we identified an association between high OGBPS score and favorable survival ( $p=0.003$; Fig. 2A). A similar association between high OGBPS score and favorable survival was observed in patients who underwent optimal $(\mathrm{p}=0.002)$ and suboptimal (approaching significance, $\mathrm{p}=0.07$ ) cytoreduction (Fig. 2B). Most importantly, OVCA patients with a high OGBPS score who underwent suboptimal cytoreduction had a survival superior to patients with a low OGBPS score who underwent optimal cytoreduction $(\mathrm{p}=0.003)$. Interestingly, patients who demonstrated a CR to primary platinum-based therapy but had a high OGBPS score had superior survival compared with those patients who demonstrated a CR but had a low OGBPS score (p=0.003) (Fig. 2C). Patients who had an IR to primary therapy had no difference in survival associated with tumor OGBPS score $(p=0.653)$ (Fig. 2D). When evaluated with cytoreductive status, grade, and age, the OGBPS score was an independent variable associated with survival $(\mathrm{p}<0.001)$.

No associations with survival were observed for the first PCA score for the Role of Nek in cell cycle regulation (59 genes, $\mathrm{p}=0.3107$ ) or the Antiviral actions of Interferons pathway (66 genes, $\mathrm{p}=0.5411)$.

\section{Discussion}

In this analysis, we applied an in vitro and in vivo genome-wide approach to define the molecular underpinnings of OVCA gemcitabine sensitivity. We identified genes and molecular signaling pathways associated with OVCA sensitivity to gemcitabine and, in doing so, have identified the OGBPS to be associated with in vitro gemcitabine response and also overall survival from OVCA. 
Table II. Genes associated with in vitro gemcitabine chemoresistance.

\begin{tabular}{|c|c|c|c|c|}
\hline Probe set ID & Gene name & Gene description & Score & p-value \\
\hline ENST00000376242_at & PSORS1C3 & PSORS1C3, AB023059.1 & 0.785 & $1.22 \mathrm{E}-09$ \\
\hline AK123047_a_at & $\mathrm{NR} 3 \mathrm{C} 2$ & NR3C2, MGC133092, MLR, MR, MCR & 0.749 & $1.72 \mathrm{E}-08$ \\
\hline ENST00000366558_a_at & KMO & KMO, dJ317G22.1 & 0.728 & $6.82 \mathrm{E}-08$ \\
\hline NM_152772_at & TCP11L2 & t-complex 11 (mouse) like 2 & 0.700 & $3.61 \mathrm{E}-07$ \\
\hline NM_003890_at & FCGBP & Human IgG Fc binding protein & 0.688 & $6.62 \mathrm{E}-07$ \\
\hline NM_021936_at & PAPPA2 & Pregnancy-associated plasma preproprotein-A2 & 0.680 & $9.89 \mathrm{E}-07$ \\
\hline NM_139173_s_at & NHEDC1 & $\mathrm{Na}^{+} / \mathrm{H}^{+}$exchanger domain CG10806-like & 0.676 & $1.25 \mathrm{E}-06$ \\
\hline NM_152888_s_at & COL22A1 & Collagen, type XXII, alpha 1 & 0.656 & $3.29 \mathrm{E}-06$ \\
\hline NM_016242_at & EMCN & Endomucin, endomucin-2 & 0.654 & $3.60 \mathrm{E}-06$ \\
\hline AL133118_at & MAPKSP1 & MAPKSP1, MAPBP, MP1, MAP2K1IP1 & 0.638 & 7.26E-06 \\
\hline NM_030923_s_at & TMEM163 & Transmembrane protein 163 & 0.636 & $8.00 \mathrm{E}-06$ \\
\hline NM_024013_at & IFNA1 & IFNA1, IFL, IFN, IFN- $\alpha$, IFNA13, IFN $\alpha$-D, LeIF D & 0.631 & $9.60 \mathrm{E}-06$ \\
\hline NM_199235_at & COLEC11 & Collectin sub-family member 11 & 0.626 & $1.18 \mathrm{E}-05$ \\
\hline NM_003585_at & $\mathrm{DOC} 2 \mathrm{~B}$ & Double $\mathrm{C} 2$-like domains, $\beta$ & 0.620 & $1.55 \mathrm{E}-05$ \\
\hline NM_005472_at & KCNE3 & Cardiac voltage-gated K channel accessory & 0.618 & $1.65 \mathrm{E}-05$ \\
\hline NM_194309_at & C21orf125 & C21orf125, PRED49, FLJ38036 & 0.618 & $1.67 \mathrm{E}-05$ \\
\hline ENST00000260323_at & UNC13C & unc-13 homolog C & 0.616 & $1.82 \mathrm{E}-05$ \\
\hline ENST00000234725_at & TMEM48 & Transmembrane protein 48 & -0.612 & $2.09 \mathrm{E}-05$ \\
\hline NM_198058_at & ZNF266 & Zinc finger protein 266 & -0.603 & $3.06 \mathrm{E}-05$ \\
\hline AW510703_at & SLC15A4 & Solute carrier family 15 , member 4 & 0.601 & $3.20 \mathrm{E}-05$ \\
\hline NM_020119_at & ZC3HAV1 & Zinc finger antiviral protein & -0.597 & $3.76 \mathrm{E}-05$ \\
\hline NM_019104_s_at & LIN37 & lin-37 homolog & 0.596 & $3.91 \mathrm{E}-05$ \\
\hline NM_022774_at & DEM1 & DEM1, FLJ11445, FLJ13183, FLJ21144, C1orf176 & -0.596 & $3.92 \mathrm{E}-05$ \\
\hline AA723953_at & SGCD & Sarcoglycan, delta (35 kDa dystrophin-associated glycan) & 0.591 & 4.82E-05 \\
\hline NM_012253_s_at & TKTL1 & Transketolase-like 1 & 0.590 & 4.87E-05 \\
\hline NM_175613_a_at & CNTN4 & Axonal cell adhesion molecule contactin 4 & 0.590 & 4.97E-05 \\
\hline NM_006198_at & PCP4 & Purkinje cell protein 4 & 0.589 & $5.01 \mathrm{E}-05$ \\
\hline NM_012391_at & SPDEF & Human prostate specific Ets, PDEF & 0.588 & $5.25 \mathrm{E}-05$ \\
\hline AK124251_at & LHFPL3 & LHFP-like protein 3 & 0.586 & $5.65 \mathrm{E}-05$ \\
\hline AK024279_a_at & WIPI2 & WD repeat domain, phosphoinositide interacting 2 & -0.583 & $6.38 \mathrm{E}-05$ \\
\hline N25888_a_at & GDF15 & Growth differentiation factor 15 & 0.581 & $6.72 \mathrm{E}-05$ \\
\hline NM_000705_at & ATP4B & ATPase, $\mathrm{H}^{+} / \mathrm{K}^{+}$transporting, beta polypeptide & 0.578 & $7.57 \mathrm{E}-05$ \\
\hline AK097996_at & GALNT2 & Polypeptide N-acetylgalactosaminyltransferase 2 & -0.577 & $7.71 \mathrm{E}-05$ \\
\hline NM_014848_at & SV2B & Synaptic vesicle protein $2 \mathrm{~B}$ & 0.577 & $7.96 \mathrm{E}-05$ \\
\hline AL049464_at & THSD4 & Thrombospondin, type I, domain containing 4 & 0.576 & $8.03 \mathrm{E}-05$ \\
\hline BM668558_at & SART1 & Squamous cell carcinoma antigen recognized by $\mathrm{T} c$ & -0.576 & $8.23 \mathrm{E}-05$ \\
\hline CR606639_a_at & ZFP57 & Zinc finger protein 57 & 0.574 & $8.62 \mathrm{E}-05$ \\
\hline NM_018053_at & XKR8 & X Kell blood group precursor-related family & -0.574 & $8.73 \mathrm{E}-05$ \\
\hline NM_002239_at & $\mathrm{KCNJ} 3$ & Subfamily, potassium inwardly-rectifying channel J3 & 0.573 & $9.09 \mathrm{E}-05$ \\
\hline BC009808_at & NBR1 & Neighbor of BRCA1 gene 1 protein & 0.573 & $9.18 \mathrm{E}-05$ \\
\hline ENST00000360944_s_at & RBAK & RB-associated KRAB repressor & -0.572 & $9.19 \mathrm{E}-05$ \\
\hline AK023318_s_at & CBARA1 & Calcium binding atopy-related autoantigen 1 & 0.570 & $9.94 \mathrm{E}-05$ \\
\hline BQ574912_s_at & TOMM5 & TOMM5, C9orf105, RP11-263I4.1, Tom5, bA613M10.3 & -0.565 & 0.0001177 \\
\hline ENST00000361262_at & SMC5 & Structural maintenance of chromosomes 5 & -0.563 & 0.0001256 \\
\hline ENST00000369578_a_at & ZNF292 & Zinc-finger domain protein & 0.563 & 0.000126 \\
\hline BC050372_a_at & OLAH & Oleoyl-ACP hydrolase & 0.563 & 0.0001284 \\
\hline NM_172238_at & TFAP2D & Transcription factor AP-2 $\beta$ & 0.563 & 0.0001288 \\
\hline NM_134266_at & SLC26A7 & Solute carrier family 26, member 7 & 0.562 & 0.0001305 \\
\hline BC027487_at & C15orf62 & & 0.561 & 0.0001364 \\
\hline
\end{tabular}


Table II. Continued.

\begin{tabular}{|c|c|c|c|c|}
\hline Probe set ID & Gene name & Gene description & Score & p-value \\
\hline DC311076_a_at & PIP4K2A & Phosphatidylinositol-4-phosphate 5-kinase type-2 & 0.561 & 0.0001374 \\
\hline NM_006786_at & UTS2 & Human urotensin II & 0.559 & 0.0001448 \\
\hline BC036592_at & GABRB2 & Gamma-aminobutyric-acid receptor beta- 2 subunit & 0.557 & 0.0001555 \\
\hline NM_018667_at & SMPD3 & Sphingomyelin phosphodiesterase 3 & 0.554 & 0.0001718 \\
\hline NM_014717_at & ZNF536 & Zinc finger protein 536 & 0.552 & 0.000184 \\
\hline NM_014629_s_at & ARHGEF10 & Rho guanine nucleotide exchange factor 10 & -0.552 & 0.000185 \\
\hline NM_001005212_at & OR9Q1 & Olfactory receptor, family 9 , subfamily $Q$ & -0.552 & 0.0001851 \\
\hline CR603904_s_at & EIF2AK2 & Protein kinase RNA-regulated (EIF2AK1) & -0.550 & 0.0001982 \\
\hline BC050525_at & USP1 & Ubiquitin-specific processing protease 1 & -0.549 & 0.0002021 \\
\hline AK024011_at & TOE1 & Target of EGR1 & -0.547 & 0.0002146 \\
\hline NM_001037165_s_at & FOXK1 & Forkhead box K1 & -0.547 & 0.0002163 \\
\hline DW432944_at & C4orf36 & C4orf36, hypothetical protein LOC132989, MGC26744, Hs.507712 & 0.547 & 0.0002164 \\
\hline NM_001551_at & IGBP1 & Immunoglobulin-binding protein 1 & 0.546 & 0.0002189 \\
\hline BX091412_at & KLHL34 & KLHL34, kelch-like 34, MGC125650, RP11-450P7.3, FLJ34960 & -0.546 & 0.0002246 \\
\hline R37641_at & CA10 & Carbonic anhydrase-related protein 10 & 0.545 & 0.0002306 \\
\hline NM_000343_at & SLC5A1 & Human $\mathrm{Na}^{+}$/glucose cotransporter $1 \mathrm{mRNA}$ & 0.545 & 0.0002323 \\
\hline BG776661_at & C10orf104 & C10orf104, FLJ33728 & 0.543 & 0.0002473 \\
\hline BC122561_at & LIN7A & Lin-7 homolog A & 0.542 & 0.0002521 \\
\hline NM_016486_at & TMEM69 & Transmembrane protein 69 & -0.541 & 0.0002638 \\
\hline M18414_at & TRDV1 & TRDV1, hDV101S1 & 0.541 & 0.000264 \\
\hline NM_014503_at & UTP20 & UTP20, down-regulated in metastasis & -0.539 & 0.0002797 \\
\hline AY153484_at & PAX2 & Paired box gene 2 & 0.537 & 0.0002962 \\
\hline BU589560_at & CLDN12 & CLDN12, claudin 12 & 0.536 & 0.0003033 \\
\hline NM_001422_s_at & ELF5 & ELF5, ESE2, ESE-2 & 0.536 & 0.0003043 \\
\hline BC038514_a_at & DPP10 & Dipeptidyl peptidase 10 & 0.536 & 0.0003078 \\
\hline BX649183_at & IVNS1ABP & Influenza virus NS1A binding protein & 0.531 & 0.0003515 \\
\hline NM_032588_at & TRIM63 & Muscle specific ring finger protein 1 & 0.531 & 0.0003543 \\
\hline NM_153705_at & KDELC2 & KDELC2, MGC33424, KDEL (Lys-Asp-Glu-Leu) containing 2 & -0.531 & 0.0003585 \\
\hline BX647977_a_at & RNMT & Human RNA (guanine-7-)methyltransferase & 0.530 & 0.0003622 \\
\hline NM_032525_at & TUBB6 & Tubulin beta- 6 chain & -0.530 & 0.0003656 \\
\hline NM_017983_at & WIPI1 & Human WD-repeat protein interacting with phosphol & 0.530 & 0.0003692 \\
\hline NM_003101_at & SOAT1 & Sterol O-acyltransferase 1 & -0.530 & 0.0003695 \\
\hline NM_182538_at & SPNS3 & SPNS3, spinster homolog 3, MGC29671 & 0.529 & 0.0003766 \\
\hline BU730580_at & RHO & Rhodopsin & 0.528 & 0.0003879 \\
\hline AL713688_s_at & hCG_2009921 & hCG_2009921, LOC441204 & 0.527 & 0.0003942 \\
\hline NM_016426_at & GTSE1 & GTSE1, G-2 and S-phase expressed 1 & -0.526 & 0.000407 \\
\hline DB377031_x_at & PSG4 & Pregnancy specific $\beta$-1-glycoprotein 4 & 0.526 & 0.0004144 \\
\hline BC101614_a_at & WDR72 & WD repeat domain 72 & 0.523 & 0.0004451 \\
\hline BI761936_a_at & C12orf69 & & 0.522 & 0.0004594 \\
\hline NM_021808_at & GALNT9 & Polypeptide N-acetylgalactosaminyltransferase 9 & 0.521 & 0.0004817 \\
\hline NM_022127_at & SLC28A3 & Concentrative $\mathrm{Na}^{+}$-nucleoside cotransporter & 0.520 & 0.00049 \\
\hline AK098151_at & PDK4 & Pyruvate dehydrogenase kinase 4 & 0.519 & 0.0005028 \\
\hline NM_174900_at & ZFP42 & Zinc finger protein 42 & 0.519 & 0.0005127 \\
\hline BC035128_a_at & MXI1 & MAX interacting protein 1 & 0.519 & 0.0005142 \\
\hline NM_001085_at & SERPINA3 & Serine proteinase inhibitor, clade A, member 3 & 0.516 & 0.0005467 \\
\hline AL564246_at & ZNF277 & Zinc finger protein 277 & 0.516 & 0.0005478 \\
\hline NM_002813_at & PSMD9 & Proteasome $26 \mathrm{~S}$ non-ATPase subunit 9 & -0.515 & 0.0005733 \\
\hline NM_005318_at & H1F0 & H1 histone family, member 0 & -0.515 & 0.0005758 \\
\hline AL136587_at & AGPAT5 & 1-acylglycerol-3-phosphate O-acyltransferase 5 & -0.514 & 0.0005852 \\
\hline
\end{tabular}


Table II. Continued.

\begin{tabular}{|c|c|c|c|c|}
\hline Probe set ID & Gene name & Gene description & Score & p-value \\
\hline NM_015474_at & SAMHD1 & SAM domain- and HD domain-containing protein 1 & -0.514 & 0.0005942 \\
\hline AV708719_at & FAM65C & FAM65C, dJ530I15.2, FLJ00360, FLJ32230, C20orf175 & 0.513 & 0.0006068 \\
\hline AF313619_at & PAQR8 & Lysosomal membrane protein in brain 1 & 0.513 & 0.0006086 \\
\hline NM_005656_at & TMPRSS2 & Transmembrane protease, serine 2 catalytic chain & 0.513 & 0.0006113 \\
\hline CN310658_s_at & FXYD6 & FXYD domain-containing ion transport regulator 6 & 0.512 & 0.000615 \\
\hline NM_032609_s_at & COX4I2 & Cytochrome c oxidase subunit 4 isoform 2 & 0.509 & 0.0006842 \\
\hline NM_007168_at & ABCA8 & ATP-binding cassette, sub-family A member 8 & 0.507 & 0.00071 \\
\hline NM_012478_at & WBP2 & WW domain binding protein 2 & 0.507 & 0.0007162 \\
\hline AK125857_at & NUP62 & Nuclear pore glycoprotein p62 & -0.507 & 0.0007163 \\
\hline NM_000078_at & CETP & Cholesteryl ester transfer protein & 0.507 & 0.0007259 \\
\hline NM_001102610_a_at & TUBGCP5 & Tubulin, gamma complex associated protein 5 & -0.505 & 0.0007604 \\
\hline NM_005773_at & ZNF256 & Zinc finger protein 256 & -0.505 & 0.0007613 \\
\hline CB852298_at & CHORDC1 & Chord domain-containing protein 1 & -0.505 & 0.0007663 \\
\hline NM_024306_at & $\mathrm{FA} 2 \mathrm{H}$ & Fatty acid hydroxylase domain containing 1 & 0.504 & 0.0007716 \\
\hline NM_031891_a_at & $\mathrm{CDH} 20$ & Cadherin 20 & 0.503 & 0.000811 \\
\hline NM_020380_at & CASC5 & Cancer susceptibility candidate 5 & -0.502 & 0.0008354 \\
\hline NM_003417_at & ZNF264 & Zinc finger protein 264 & -0.501 & 0.000841 \\
\hline NM_018840_at & C20orf24 & C20orf24, PNAS-11, RAB5-interacting protein, RIP5 & -0.501 & 0.0008587 \\
\hline NM_021269_s_at & ZNF708 & Zinc finger protein 15 -like $1(\mathrm{KOX} 8)$ & -0.500 & 0.0008837 \\
\hline NM_020167_at & NMUR2 & Neuromedin U receptor 2 & 0.499 & 0.0008887 \\
\hline NM_001112724_at & STK32A & Serine/threonine kinase $32 \mathrm{~A}$ & 0.499 & 0.0008995 \\
\hline AK075129_s_at & RHOBTB1 & Rho-related BTB domain containing 1 & 0.498 & 0.0009097 \\
\hline ENST00000357899_a_at & ZBTB44 & Zinc finger and BTB domain containing 44 & 0.498 & 0.0009199 \\
\hline CR456455_s_at & SERHL & Serine hydrolase-like & -0.498 & 0.0009238 \\
\hline NM_080717_at & TBX5 & T-box transcription factor TBX5 & 0.498 & 0.0009268 \\
\hline BC098116_at & ABCA11P & $\begin{array}{l}\text { FLJ14297, MGC120309, MGC120310, MGC120312, MGC132744, } \\
\text { MGC138274 }\end{array}$ & -0.498 & 0.0009313 \\
\hline AK026107_a_at & RBM25 & RNA binding motif protein 25, RNA-binding motif protein 25 & -0.497 & 0.0009449 \\
\hline LIT1500_s_at & NOL5A & Nucleolar protein $5 \mathrm{~A}$ & -0.497 & 0.0009486 \\
\hline AF233261_a_at & OTOR & $\begin{array}{l}\text { OTOR, fibrocyte-derived protein, melanoma inhibitory activity-like } \\
\text { B protein }\end{array}$ & 0.497 & 0.0009601 \\
\hline CR610033_a_at & TOM1L1 & Target of Myb-like protein 1 & 0.496 & 0.0009693 \\
\hline AI144436_at & SF3A3 & Spliceosome-associated protein 61 , Splicing factor 3 A subunit 3 & -0.495 & 0.0009879 \\
\hline AB053232_at & GAL3ST3 & Galactose 3'-sulfotransferase, galactose-3-O-sulfotransferase 3 & 0.495 & 0.0009897 \\
\hline NM_206915_s_at & NGFRAP1 & NGFRAP1, BEX3, DXS6984E, HGR74, NADE, Bex & -0.495 & 0.0009941 \\
\hline
\end{tabular}

Previous efforts to define the molecular basis of gemcitabine resistance have identified molecules such as deoxycytidine kinase (dCK) (8,17-19), ribonucleotide reductase (20-22), and human equilibrative nucleoside transporter-1 (hENT1) (4,23-26). Decreased activity of dCK, which phosphorylates gemcitabine to its monophosphate form, has previously been reported to be associated with resistance to gemcitabine (8,17-19). Consistent with these data, in our analysis, we demonstrated a negative correlation between OVCA cell line mRNA expression of the $\mathrm{dCK}$ gene and increasing gemcitabine resistance (Pearson's correlation: $-0.33, p=0.05)$. Previously, overexpression of the M1 and M2 subunits of ribonucleotide reductase (RRM1 and
RRM2) has been demonstrated to be associated with gemcitabine resistance in gastrointestinal cancer cells $(27,28)$. In our analysis, we observed no association between gemcitabine resistance and expression of RRM1, although we observed an association between low levels of RRM2 expression (using median expression as a threshold) and high gemcitabine $\mathrm{IC}_{50}(\mathrm{p}<0.02)$. It is unclear why our findings are contradictory to those of Davidson et al (27); however, they may be due to differences in cancer types studied. Inhibition of hENT1 was previously reported to be associated with gemcitabine chemoresistance $(4,25)$. This correlates with our findings in which we demonstrated a negative correlation between OVCA cell line mRNA expression of the 


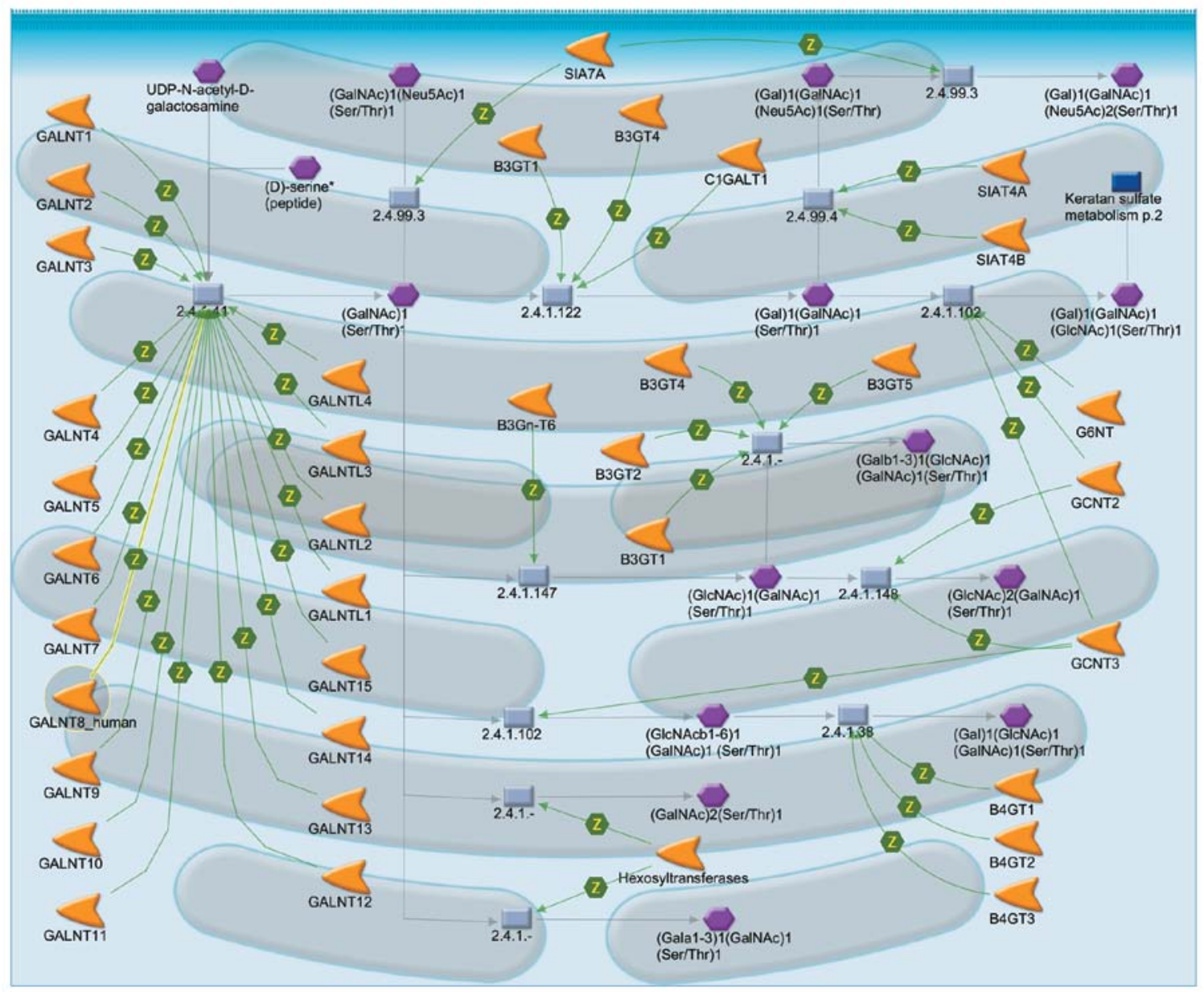

Figure 1. O-glycan biosynthesis/human version pathway.
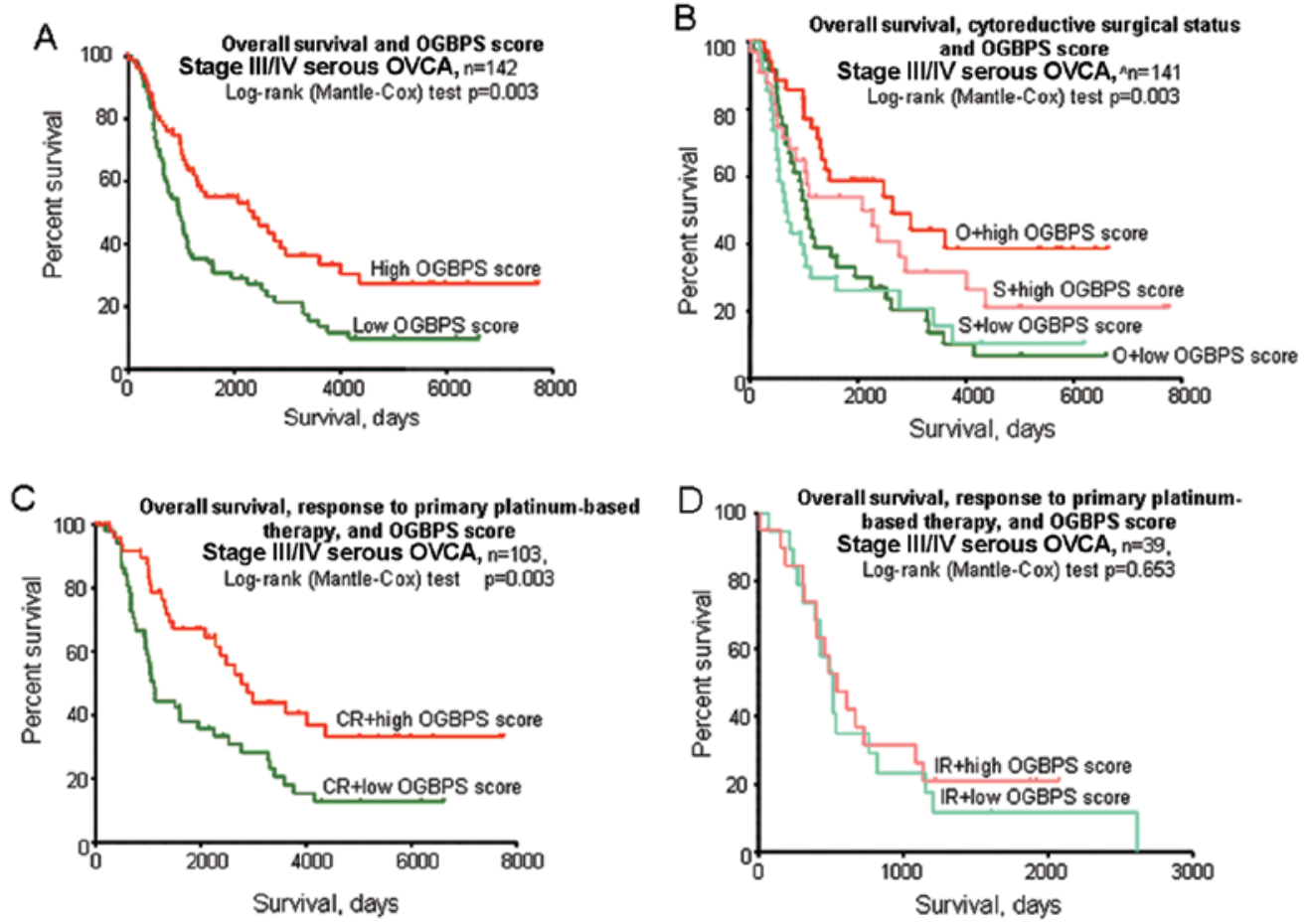

Figure 2. High OGBPS PCA score is associated with favorable clinical outcome. Kaplan-Meier curves depicting the association between OGBPS PCA score and (A) overall survival from OVCA; (B) overall survival and cytoreductive status, (C) overall survival and complete response to platinum therapy median cutoff, and

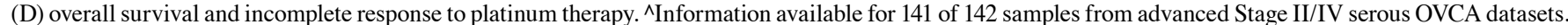
The numbers at risk are shown at the top of graphs. Log-rank test p-values indicate significance. CR, complete response; IR, incomplete response, O, optimal; S, suboptimal. 
Table III. OGBPS 34 genes.

\begin{tabular}{|c|c|c|}
\hline NM_020981_at & B3GALT1 & Beta-1,3-galactosyltransferase \\
\hline NM_003783_at & B3GALT2 & Beta-1,3-galactosyltransferase, beta-3-galt2 \\
\hline NM_003782_a_at & B3GALT4 & Beta-1,3-galactosyltransferase 4 \\
\hline NM_033171_at & B3GALT5 & GlcNAc-beta-1,3-galactosyltransferase 5, GLCT5, homolog of C \\
\hline NM_138706_at & B3GNT6 & Beta-1,3-N-acetylglucosaminyltransferase protein \\
\hline U10474_at & B4GALT1 & B4GALT1 \\
\hline NM_003780_at & B4GALT2 & B4GALT2 \\
\hline NM_003779_at & B4GALT3 & Beta4Gal-T3 \\
\hline NM_020156_at & C1GALT1 & Core 1 synthase, glycoprotein-N-acetylgalactosamine \\
\hline AW798875_at & GALNT1 & Polypeptide N-acetylgalactosaminyltransferase 1 \\
\hline NM_024564_at & GALNT10 & Polypeptide N-acetylgalactosaminyltransferase 10 \\
\hline NM_022087_at & GALNT11 & Polypeptide N-acetylgalactosaminyltransferase 11 \\
\hline AI638649_at & GALNT12 & Polypeptide N-acetylgalactosaminyltransferase 12 \\
\hline AK131195_a_at & GALNT13 & UDP-N-acetyl-alpha-D-galactosamine:polypeptide \\
\hline NM_024572_s_at & GALNT14 & Polypeptide N-acetylgalactosaminyltransferase 14 \\
\hline AK097996_at & GALNT2 & Polypeptide N-acetylgalactosaminyltransferase 2 \\
\hline BX647473_a_at & GALNT3 & Polypeptide N-acetylgalactosaminyltransferase 3 \\
\hline NM_003774_at & GALNT4 & Polypeptide N-acetylgalactosaminyltransferase 4 \\
\hline BX097451_s_at & GALNT5 & Polypeptide N-acetylgalactosaminyltransferase 5 \\
\hline BU542820_at & GALNT6 & Polypeptide N-acetylgalactosaminyltransferase 6 \\
\hline NM_017423_at & GALNT7 & Polypeptide N-acetylgalactosaminyltransferase 7 \\
\hline BM719843_a_at & GALNT8 & $\mathrm{N}$-acetylgalactosaminyltransferase 8 \\
\hline NM_021808_at & GALNT9 & Polypeptide N-acetylgalactosaminyltransferase 9 \\
\hline NM_020692_at & GALNTL1 & Polypeptide N-acetylgalactosaminyltransferase 16 \\
\hline BC030625_at & GALNTL2 & Polypeptide N-acetylgalactosaminyltransferase 13 \\
\hline NM_198516_at & GALNTL4 & UDP-N-acetyl-alpha-D-galactosamine \\
\hline NM_145292_at & GALNTL5 & UDP-N-acetyl-alpha-D-galactosamine \\
\hline NM_001490_at & GCNT1 & Beta-1,6-N-acetylglucosaminyltransferase 1 \\
\hline NM_145649_s_at & GCNT2 & Beta-1,6-N-acetylglucosaminyltransferase 2 \\
\hline NM_004751_at & GCNT3 & Beta1,6-N-acetylglucosaminyltransferase 3 \\
\hline CR619813_at & ST3GAL1 & 3-Sialyltransferase,Gal-NAc6S \\
\hline AK127322_at & ST3GAL2 & Beta-galactoside alpha-2,3-sialytransferase \\
\hline NM_018414_at & ST6GALN & 6-Sialyltransferase I alpha-N-acetylgalactosaminide alpha-2 \\
\hline BC067524_a_at & WBSCR17 & $\begin{array}{l}\text { Polypeptide N-acetylgalactosaminyltransferase, } \\
\text { Williams-Beuren syndrome chromosome region } 17\end{array}$ \\
\hline
\end{tabular}

hENT1 gene and increasing gemcitabine resistance (Pearson's correlation: $-0.3, \mathrm{p}=0.06$ ).

The process of glycosylation involves the enzymatic addition of carbohydrates to proteins or lipids and is the most common form of post-translational modification. Three categories of protein-linked glycans exist, including those linked to the amide group of asparagine (N-linked), those linked to the hydroxyl group of serine, threonine, or hydroxylysine 3 (O-linked), and those linked to a carboxyl group of tryptophan (C-linked) (29). The main pathway for complex O-glycan biosynthesis is located in the endoplasmic reticulum and Golgi compartments, restricting glycosylation largely to the endoplasmic reticulum, Golgi, lysosomal, plasma membrane, and secretory proteins, with the exception of nuclear and cytosolic proteins, which can be modified with a single O-linked GlcNAc (30). O-glycans have been reported to have a broad range of functions in protein structure and stability, immunity, receptor-mediated signaling, non-specific protein interactions, modulation of the activity of enzymes and signaling molecules, and protein expression and processing $(30,31)$. Although these biological roles range in importance, they can be critical for development, growth, function, and survival. Moreover, a specific O-glycan may influence a range of functions at different locations and times within an organism (31). Previously, limited access to endoglycosidases to cleave intact $\mathrm{O}$-glycans from their protein backbone, as well as the extreme diversity of their structures, has limited research relative to study of $\mathrm{N}$-linked glycan pathway-linked diseases (historically considered the congenital disorders of 
glycosylation). More recently, in human cancers, O-glycans have been shown to play important roles in cancer cell attachment, signaling, invasion (32-35), and survival in the bloodstream. Inhibition of the O-glycan pathway in colorectal cancer cell lines has been shown to inhibit cell growth and induce apoptosis (36). Down-regulation of the N-glycan biosynthesis pathway was also reported to be associated with chemoresistance in cholangiocarcinoma cell lines (37).

To date, we are unaware of any reports suggesting that the O-glycan pathway influences OVCA cell response to therapeutic interventions or overall survival. In this study, expression of the O-glycan pathway (quantified by a OGBPS score) was associated with OVCA overall survival when we analyzed: a) all patients with OVCA, b) patients who underwent optimal OVCA surgical cytoreduction, and c) patients who experienced a CR to primary surgery plus platinum-based therapy. The association between OGBPS score and overall survival for patients who underwent suboptimal surgical cytoreduction did not reach statistical significance $(\mathrm{p}=0.07)$, and no association was identified in patients who experienced an IR to primary surgery plus platinum-based therapy. When evaluated with cytoreductive status, grade, and age, the OGBPS score was an independent variable associated with survival $(\mathrm{p}<0.001)$. The explanation for the associations between OGBPS and OVCA survival is likely complex. Although in this study we identified the O-glycan pathway by its association with in vitro gemcitabine sensitivity, we do not believe that the impact of the pathway on overall survival is driven by its effect of gemcitabine sensitivity. In fact, high OGBPS score was associated with resistance to gemcitabine, yet showed a more favorable outcome for patients with OVCA. As noted above, O-glycans are known to influence cancer cell attachment, signaling, invasion, and survival in the bloodstream (32-35). It is likely that the effect on OVCA clinical outcome is associated with one or more of these important oncologic processes. It will therefore be essential in future studies to investigate associations between OGBPS score, activity of individual members of the O-glycan pathway, and OVCA cell phenotypic behavior.

Our discovery of associations between O-glycan pathway expression and gemcitabine sensitivity and patient survival is novel. These findings potentially have substantial implications for future clinical management of patients with OVCA. In the future, empiric-based treatment decision-making must be replaced with a more tailored strategy that stratifies patients based on their molecular fingerprints. Such an approach will identify those patients with the 'highest risk' disease, those who may benefit from additional pathway-targeted therapy added to standard of care cytotoxic regimens, and potentially those who may (or may not) benefit from aggressive surgical interventions.

\section{Acknowledgements}

Opinions, interpretations, conclusions, and recommendations are those of the authors and are not necessarily endorsed by the funding agencies. We thank Rasa Hamilton (Moffitt Cancer Center) for editorial assistance. We also like to thank Merck pharmaceuticals for their contributions. This study was supported in part by the Hearing the Ovarian Cancer Whisper, Jacquie Liggett Foundation, the National Cancer Institute Grant
R21 CA-110499-01A2, the Ocala Royal Dames for Cancer Research Inc., the Phi Beta Psi Sorority, the Ovarian Cancer Research Fund, and the US Army Medical Research and Materiel Command under award no. DAMD17-02-2-0051.

\section{References}

1. Gadducci A, Sartori E, Maggino T, et al: Analysis of failures after negative second-look in patients with advanced ovarian cancer: an Italian multicenter study. Gynecol Oncol 68: 150-155, 1998.

2. McGuire WP, Hoskins WJ, Brady MF, et al: Cyclophosphamide and cisplatin compared with paclitaxel and cisplatin in patients with stage III and stage IV ovarian cancer. N Engl J Med 334: 1-6, 1996

3. Ozols RF: The current role of gemcitabine in ovarian cancer. Semin Oncol 28: 18-24, 2001.

4. Mackey JR, Mani RS, Selner M, et al: Functional nucleoside transporters are required for gemcitabine influx and manifestation of toxicity in cancer cell lines. Cancer Res 58: 4349-4357, 1998.

5. Distefano M,Ferlini C, De Vincenzo R, Gaggini C, Mancuso S and Scambia G: Antagonistic effect of the combination gemcitabine/ topotecan in ovarian cancer cells. Oncol Res 12: 355-359, 2000.

6. Peters GJ, Bergman AM, Ruiz van Haperen VW, Veerman G, Kuiper CM and Braakhuis BJ: Interaction between cisplatin and gemcitabine in vitro and in vivo. Semin Oncol 22: 72-79, 1995.

7. Ruiz van Haperen VW, Veerman G, Boven E, Noordhuis P Vermorken JB and Peters GJ: Schedule dependence of sensitivity to 2',2'-difluorodeoxycytidine (Gemcitabine) in relation to accumulation and retention of its triphosphate in solid tumour cell lines and solid tumours. Biochem Pharmacol 48: 1327-1339, 1994.

8. Ruiz van Haperen VW, Veerman G,Eriksson S, et al: Development and molecular characterization of a 2',2'-difluorodeoxycytidineresistant variant of the human ovarian carcinoma cell line A2780. Cancer Res 54: 4138-4143, 1994.

9. Ruiz van Haperen VW, Veerman G, Eriksson S, Stegmann AP and Peters GJ: Induction of resistance to 2',2'-difluorodeoxycytidine in the human ovarian cancer cell line A2780. Semin Oncol 22: 35-41, 1995.

10. Van Moorsel CJ, Veerman G, Bergman AM, et al: Combination chemotherapy studies with gemcitabine. Semin Oncol 24 (Suppl 7): 17-23, 1997.

11. Braakhuis BJ, Ruiz van Haperen VW, Boven E, Veerman G and Peters GJ: Schedule-dependent antitumor effect of gemcitabine in in vivo model system. Semin Oncol 22: 42-46, 1995.

12. Friedlander M, Millward MJ, Bell D, et al: A phase II study of gemcitabine in platinum pre-treated patients with advanced epithelial ovarian cancer. Ann Oncol 9: 1343-1345, 1998.

13. Lund B, Hansen OP, Theilade K, Hansen M and Neijt JP: Phase II study of gemcitabine (2',2'-difluorodeoxycytidine) in previously treated ovarian cancer patients. J Natl Cancer Inst 86: 1530-1533, 1994.

14. Markman M, Webster K, Zanotti K, Kulp B, Peterson G and Belinson J: Phase 2 trial of single-agent gemcitabine in platinumpaclitaxel refractory ovarian cancer. Gynecol Oncol 90: 593-596, 2003.

15. Chen DT, Nasir A, Culhane A, et al: Proliferative genes dominate malignancy-risk gene signature in histologically-normal breast tissue. Breast Cancer Res Treat 119: 335-346, 2010.

16. Marchion DC, Cottrill HM, Xiong Y, et al: BAD phosphorylation determines ovarian cancer chemo-sensitivity and patient survival. Clin Cancer Res 17: 6356-6366, 2011.

17. Galmarini CM, Mackey JR and Dumontet C: Nucleoside analogues: mechanisms of drug resistance and reversal strategies. Leukemia 15: 875-890, 2001.

18. Jordheim L, Galmarini CM and Dumontet C: Drug resistance to cytotoxic nucleoside analogues. Curr Drug Targets 4: 443-460, 2003.

19. Van der Wilt CL, Kroep JR, Bergman AM, et al: The role of deoxycytidine kinase in gemcitabine cytotoxicity. Adv Exp Med Biol 486: 287-290, 2000.

20. Cory JG and Sato A: Regulation of ribonucleotide reductase activity in mammalian cells. Mol Cell Biochem 53-54: 257-266, 1983.

21. Thelander L and Berg P: Isolation and characterization of expressible cDNA clones encoding the M1 and M2 subunits of mouse ribonucleotide reductase. Mol Cell Biol 6: 3433-3442, 1986. 
22. Zhou BS, Tsai P, Ker R, et al: Overexpression of transfected human ribonucleotide reductase M2 subunit in human cancer cells enhances their invasive potential. Clin Exp Metastasis 16: 43-49, 1998

23. Garcia-Manteiga J, Molina-Arcas M, Casado FJ, Mazo A and Pastor-Anglada M: Nucleoside transporter profiles in human pancreatic cancer cells: role of hCNT1 in 2',2'-difluorodeoxycytidine-induced cytotoxicity. Clin Cancer Res 9: 5000-5008, 2003.

24. Mackey JR, Yao SY, Smith KM, et al: Gemcitabine transport in xenopus oocytes expressing recombinant plasma membrane mammalian nucleoside transporters. J Natl Cancer Inst 91: 1876-1881, 1999.

25. Rauchwerger DR, Firby PS, Hedley DW and Moore MJ : Equilibrative-sensitive nucleoside transporter and its role in gemcitabine sensitivity. Cancer Res 60: 6075-6079, 2000.

26. Ritzel MW, Ng AM, Yao SY, et al: Recent molecular advances in studies of the concentrative $\mathrm{Na}^{+}$-dependent nucleoside transporter (CNT) family: identification and characterization of novel human and mouse proteins (hCNT3 and mCNT3) broadly selective for purine and pyrimidine nucleosides (system cib). Mol Membr Biol 18: 65-72, 2001.

27. Davidson JD, Ma L, Flagella M, Geeganage S, Gelbert LM and Slapak CA: An increase in the expression of ribonucleotide reductase large subunit 1 is associated with gemcitabine resistance in non-small cell lung cancer cell lines. Cancer Res 64: 3761-3766, 2004.

28. Jung CP, Motwani MV and Schwartz GK: Flavopiridol increases sensitization to gemcitabine in human gastrointestinal cancer cell lines and correlates with down-regulation of ribonucleotide reductase M2 subunit. Clin Cancer Res 7: 2527-2536, 2001.
29. Hofsteenge J, Muller DR, De Beer T, Loffler A, Richter WJ and Vliegenthart JF: New type of linkage between a carbohydrate and a protein: C-glycosylation of a specific tryptophan residue in human RNase Us. Biochemistry 33: 13524-13530, 1994.

30. Wells L and Hart GW: O-GlcNAc turns twenty: functional implications for post-translational modification of nuclear and cytosolic proteins with a sugar. FEBS Lett 546: 154-158, 2003.

31. Varki A: Biological roles of oligosaccharides: all of the theories are correct. Glycobiology 3: 97-130, 1993.

32. Fuster MM, Brown JR, Wang L and Esko JD: A disaccharide precursor of sialyl Lewis X inhibits metastatic potential of tumor cells. Cancer Res 63: 2775-2781, 2003.

33. Gabius HJ: Cell surface glycans: the why and how of their functionality as biochemical signals in lectin-mediated information transfer. Crit Rev Immunol 26: 43-79, 2006.

34. Huet G, Gouyer V, Delacour D, et al: Involvement of glycosylation in the intracellular trafficking of glycoproteins in polarized epithelial cells. Biochimie 85: 323-330, 2003.

35. Ulloa F and Real FX: Benzyl-N-acetyl-alpha-D-galactosaminide induces a storage disease-like phenotype by perturbing the endocytic pathway. J Biol Chem 278: 12374-12383, 2003.

36. Patsos G, Hebbe-Viton V, Robbe-Masselot C, et al: O-glycan inhibitors generate aryl-glycans, induce apoptosis and lead to growth inhibition in colorectal cancer cell lines. Glycobiology 19: 382-398, 2009.

37. Sato J, Kimura T, Saito T, et al: Gene expression analysis for predicting gemcitabine resistance in human cholangiocarcinoma. J Hepatobiliary Pancreat Sci 18: 700-711, 2011. 